\title{
The Virtual Transformational Leadership Development Experience: Creating a Classroom of the Future
}

\author{
Dr. Tom C. Hogan ${ }^{1}$, Sean Gallagher ${ }^{2}$, Nathan Ousey ${ }^{3}$, Ray Schmitt ${ }^{4}$ \\ ${ }^{1}$ Professor of Practice in Human Resource Management, School of Labor and Employment Relations Scholar-in-Residence, \\ Center for the Performing Arts, College of Arts and Architecture, Penn State University, Pennsylvania, USA \\ ${ }^{2}$ Undergraduate Student, Penn State University, Pennsylvania, USA \\ ${ }^{3}$ Research Assistant, College of Liberal Arts, Penn State University, Pennsylvania, USA \\ ${ }^{4}$ Public Relations Specialist, Penn State University, Pennsylvania, USA \\ *Correspondence: Dr. Tom C. Hogan, tch12@psu.edu
}

\begin{abstract}
In response to a new set of challenges facing universities today-developing the next generation of leaders while integrating existing and emerging technologies to transform learning spaces - a team at Penn State University conducted research that culminated in the Virtual Transformational Leadership Development (VTLD) Experience, a course that addresses these challenges in a virtual format. The research examined the contribution of the arts, in conjunction with immersive technologies, towards promoting student engagement. Student interviews indicated that the creation of high impact practices revolving around the arts ultimately generates a memorable and involved experience that more frequently includes topics of social relevancy such as racial equity. Furthermore, the pairing of these methods with relevant and emerging technologies, such as artificial intelligence (AI) and virtual reality (VR), effectively creates an educational experience that more personally suits a student's needs in this type of transformational course.
\end{abstract}

Keywords: Student engagement, Leadership, Social justice, Transformation, Technology, Arts

General Terms: Individual study, Interview collection, Secondary research

\section{ARTICLE INFORMATION}

Author(s): Dr. Tom C. Hogan ${ }^{1}$, Sean Gallagher ${ }^{2}$, Nathan Ousey ${ }^{3}$, Ray Schmitt ${ }^{4}$

Received: 11 May, 2021; Accepted: 15 June, 2021; Published: 30 June, 2021; e-ISSN: 2347-4696;

Paper Id: BMN-IJBMR-2021-39;

Citation: $10.37391 /$ IJBMR.090212

Webpage-link:

https://ijbmr.forexjournal.co.in/archive/volume-9/ijbmr-090212.html

\section{INTRODUCTION}

Higher education is constantly changing and evolving to educate students, prepare learners for citizenship, and to better serve society. Universities' response to the global COVID-19 pandemic serves as a concrete example of how quickly higher education can pivot and respond to changes on a larger and connected scale. Transitioning from in-person classes to remote virtual classes was accomplished in a short period of time, leaving educators with the task of determining how to sustain the benefits that came, some unexpectedly, with these changes [1]. Moving forward from this period, a remaining challenge facing higher education involves how faculty can help to develop the next generation of leaders with the skill set and mindset to thrive in a diverse, increasingly polarized, and constantly changing world. A related challenge is how existing and emerging technologies can be embedded into pedagogy and curriculum to create personalized and transformative learning experiences, drive student learning outcomes, and promote student engagement and engaged scholarship (i.e., out-of-classroom learning).

With these challenges in mind, undergraduate researchers from Penn State's School of Labor and Employment Relations hypothesized that by leveraging the arts in a virtual setting, educators in higher education can maintain and promote student engagement. This study, titled Creating Transformative Experiences: The Art of Student Engagement and Engaged Scholarship, sought to understand the potential contributions of the arts in inspiring students to become psychologically involved, connected to, and passionate about learning.

\subsection{Literature Review}

In Student Engagement in Higher Education, Harper and Quaye [2] identify a crucial definition: "student engagement is simply characterized as participation in educationally effective practices, both inside and outside the classroom which leads to a range of measurable outcomes," identifying that engagement is the single most significant predictor of student persistence. The current study aims to fully acknowledge this significance and definition by determining the potential role of the arts and virtual learning in maintaining student persistence.

In a paper discussing artistic ways of engaging students, Irving [3] presents research surrounding a series of films in which dance is used to demonstrate statistical concepts in psychology. Rather than intending to substitute for lectures, Irving explains that these films were designed to help students think about statistical concepts in "different and memorable" ways. These films received noteworthy feedback in engaging students by many educators." This research presents an opportunity for further exploration at the college level for a learning and engagement tool that could be a useful approach to learning when applied with an appropriate balance between 
this material and the virtual learning environment.

In a study from Chistopoulos, et al. [4], the impact of student engagement using virtual worlds and environments is measured through a priori and a posteriori survey. They concluded that the use of virtual worlds as a teaching tool can positively impact learner engagement, and that simultaneously using physical and virtual locations allowed the learners to eliminate the weaknesses of each environment. The current research aims to further examine the validity of virtual environments as well as test the benefits of incorporating artsbased modules into virtual reality.

\subsection{VTLD Experience}

Using this research as a basis, a team comprised of faculty, Teaching and Learning with Technology staff, and undergraduate students at Penn State University is creating and testing an innovative approach to developing the next generation of leaders to serve as agents of change in pursuit of a more civil, equitable and just workplace and society. The Virtual Transformational Leadership Development (VTLD) Experience is an extension of the research, teaching, and international advisory work on racial equity and social justice of Dr. Hogan, PI., who served as a Scholar, Engagement Academy $(2019$ - 2021) and as a Teaching and Learning with Technology (TLT) Faculty Fellow (2020 - 2021).

\section{LEVERAGING RESEARCH}

The VTLD Experience leverages the findings of its associated study, such as the importance of incorporating High Impact Practices (HIPs), to create transformational and interactive learning experiences for undergraduate students. HIPs refer to experiences that more heavily involve students and require them to reflect on their engagement with specific materials, such as being exposed to and attending an arts performance and interacting with artists afterwards. Through the integration of existing and emerging technologies, this learning experience will provide lesson modules that include viewing and reflecting on works of art, such as visual art, performances, and other forms of creative expression. The research demonstrates how the arts engage students in a variety of subjects, such as mindfulness practices to develop mindful leadership skills and lessons about global diversity, equity, inclusion, and belonging. Through exposure to different perspectives to promote cognitive diversity, this learning experience seeks to nurture the whole student thoughts, feelings, body - by developing skill sets and mindsets to increase their senses of curiosity, empathy, emotional and cultural intelligence, and to develop resilience.

Figure 1 represents a research statistic that motivates the course's prioritization of integrating High Impact Practices with first-year students' education. Only an average of fifty percent of first years are engaged in at least one HIP, and rarely are they involved in two or more. By leveraging the arts and immersive technology with new HIPs for these students early in their higher education career, educators can ensure students have a more involved experience throughout their entire duration at universities.

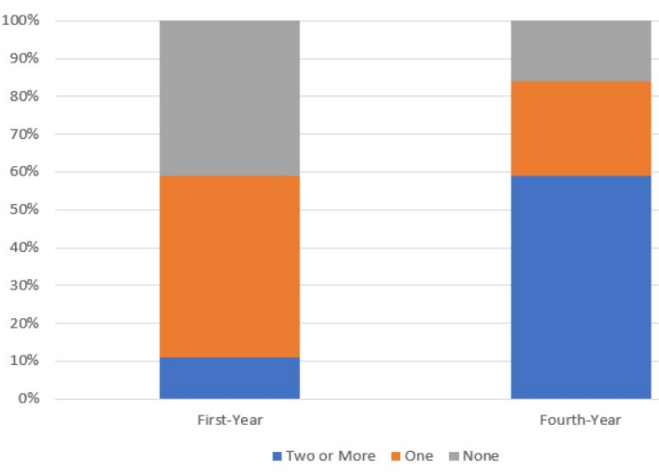

Note: All counts include participation in service-learning, learning community, and research with faculty. The fourth-year counts also include internship and field experience, study abroad, and culminating senior experience.

Figure 1: Percentage of students experiencing "One", "Two or more", or "None" high impact practices.

The current study conducted video interviews with students attending arts-based programming in 2020. After witnessing a performance by Step Afrika! - the first professional company devoted to the tradition of stepping - participants were given the opportunity to interact with the performers with questions concerning their dance's message and cultural roots. In interviews held afterwards, participants noted their pleasure in watching the performance and interacting with the performing, several of them noting how this engagement helped them to understand more about the diverse cultures showcased in the programming. Step Afrika! also held a virtual performance in September 2020 with the same variables in their performance and interaction with participants, evoking the same responses and reactions of the students involved. This part of the study methodology helped to show virtual programming as a successful alternative to in-person events.

\subsection{Technology and Arts in Promoting Engagement}

Transformative learning spaces such as The VTLD Experience leverage technology - artificial intelligence (AI), machine learning (ML), augmented and virtual reality (AR and VR), and gamification - to promote student engagement, encourage deep thinking, and drive student learning outcomes. The study by Christopoulos, et al. [4] (See 1.1 Literature Review) demonstrates the validity of such environments as emerging modes of learning, its findings only strengthened after the COVID-19 transition to online education. The VTLD Experience seeks to leverage AR and VR as an enhanced medium through which students can experience the arts, develop new skill sets and mind-sets and develop global leadership skills.

In another study used as secondary research, "The Effectiveness of Virtual and Augmented Reality in Health Sciences and Medical Anatomy," participating students were taught identical lessons on the anatomy of the human skull while using three modes of delivery: VR, AR, and 3D tablets. The results of the report concluded that VR, AR, and 3D tablets were effective teaching tools, and specifically that "VR 
and AR provide additional intrinsic benefits, such as increased student engagement, interactivity, and enjoyment" [5]. These relevant findings help inform the key findings of the current study, as "engagement, interactivity, and enjoyment" align with the values repeated in student interviews related to arts programming. As such, arts-based learning and virtual environments evoke the same classifications of student engagement and can be used in conjunction with one another to amplify this effect.

The VTLD Experience also leverages research on AI to create a virtual intelligent coach, available on demand, to support student growth and development and to respond to frequently asked questions. Currently, there are several institutions of higher education using AI to improve learning; colleges such as Staffordshire University in the U.K and Georgia Tech in the U.S have begun using chatbots that provide 24/7 help and answers to students. AI-enabled technology has also shown to promote a more effective learning experience. Esme Learning Solutions is one example; the digital learning platform incorporates AI-enabled tools based around optimizing human performance and enhancing digital collaboration into their sixweek-long courses based on 15 years of peer-reviewed cognitive and neuroscience research [6]. Approximately $90 \%$ of students found the course content engaging, and when compared to other learning companies, Esme Learning students saw $23 \%$ higher grades over learners who simply read and viewed the materials [6].

\subsection{The Human Experience and Social Justice}

While these technologies can increase engagement among students and lead to better student learning outcomes, one must consider the necessity of incorporating the human experience and adopting a human-centric approach to AI to minimize algorithmic bias when using these tools. Deloitte, a large consulting company, has published findings regarding the Human Experience, citing that it is replacing the customer experience across organizations [7]. People want to be treated like humans and to be able to connect with others on a personal level, forming real relationships.

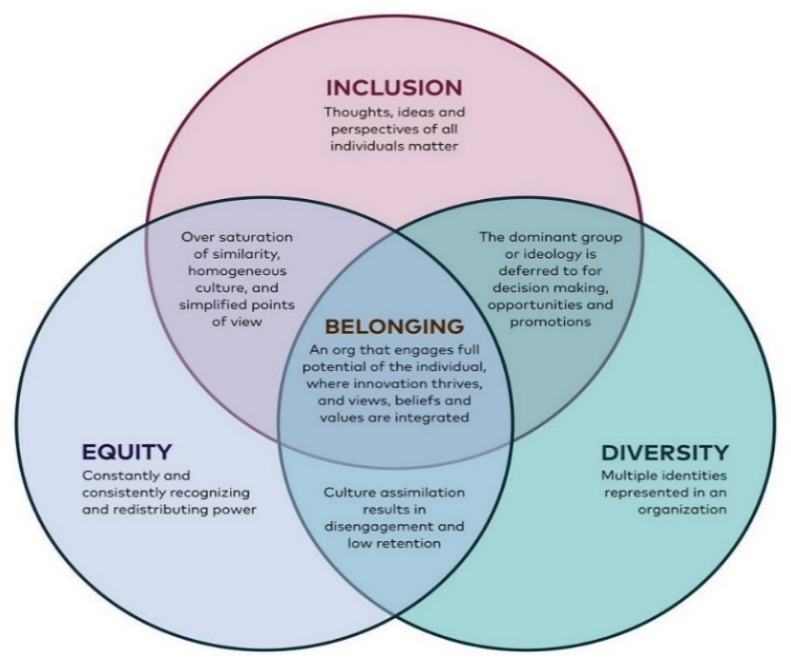

Figure 2: Model of Belonging.
With this reasoning in mind, The VTLD Experience recognizes that virtual arts programming creates avenues for sharing common human experiences at the university level, where students and the community can attend events, meet people, and gain knowledge and exposure to perspectives they might not get to experience during a normal day of classes and work. The VTLD Experience also considers the human experience by seeking to create a greater sense of belonging in students. A guiding principle of The VTLD Experience is that diversity is a fact, inclusion is a choice, equity is an action, and belonging is an outcome, as organized in a Venn Diagram model by Krys Burnette [8] (Figure 2).

\subsection{Key Findings}

Through aforementioned data collection, student interviews, and secondary research, the current study's findings support the claim that usage of the arts in a virtual setting promotes student engagement and fosters a greater sense of belonging amongst students. As referenced in Section 2, student interviews confirm arts programming as uniquely effective in keeping participants engaged in High Impact Practices. Secondary research points towards immersive technologies such as Virtual Reality as capable of promoting a similar sense of engagement and "enjoyment" and supplements the arts experience. Furthermore, Artificial Intelligence increases student involvement by meeting their needs on an individual basis through a virtual coach. Student interviews also highlighted the importance of top-down student engagement, wherein arts programming is deemed more effective by students if engaged with by the higher levels of administration first, therefore dismissing any doubts of these activities' effectiveness. Interviews also recognize the benefits of artsbased modules in exposing participants to other perspectives and cultures not of their own.

These findings build upon the work of previous research by making a connection between the effectiveness of the arts in promoting engagement [3] with the potential for its use in higher education through virtual means [4]. By proving the effectiveness of HIPs centered around the arts in higher education, the current study lays out the groundwork for transformative learning spaces such as the VTLD Experience.

\section{CUMULATIVE VISION}

Combining teaching and learning with existing and emerging technologies, The VTLD Experience, in conjunction with the human experience, generates a vision for the classroom of the future. These technologies enable learning spaces where students and faculty can take advantage of virtual arts programming to "attend" events around the world that would otherwise be inaccessible, such as museums, works of art, and live performances. This is an example of what the classroom of the future might look like, and we are actively pursuing this vision. We will be offering a pilot of The VTLD Experience fall 2021 and spring 2022 semesters at Penn State. Future enhancements of the experience will incorporate additional technologies and applications. 
This research allows us to rethink and reimagine the future and transcendence of classroom spaces into virtual learning spaces. Institutions of higher education are using High Impact Practices and existing and emerging technologies to improve the student learning experience and drive student learning outcomes. It is our duty and obligation as ethical leaders and educators to create more welcoming and inclusive teaching and learning environments. This includes serving the needs of underrepresented, underserved, and marginalized student populations. Ultimately, The VTLD Experience is designed to help create a more civil, just, and equitable future for all.

\section{REFERENCES}

[1] Nworie, J. (2021). Beyond COVID-19: What's Next for Online Teaching and Learning in Higher Education? EDUCAUSE Review.

[2] Harper, S. R., \& Quaye, S. J. (2015). Student engagement in higher education: theoretical perspectives and practical approaches for diverse populations. New York, USA: Routledge.
[3] Irving, L.T. (2015). Teaching Statistics Using Dance and Movement. Frontiers in Psychology, 6, 50.

[4] Christopoulos, A., Conrad, M. \& Shukla, M. (2018). Increasing student engagement through virtual interactions: How? Virtual Reality 22, 353-369.

[5] Moro, C., Štromberga, Z., Raikos, A. and Stirling, A. (2017). The effectiveness of virtual and augmented reality in health sciences and medical anatomy. American Association of Anatomists, 10: 549-559.

[6] Butkovik, M. (2021). Esme Learning Raises \$7.5 Million in Series A Funding for AI-Enabled Digital Learning Platform. Forbes.

[7] Dunlop, A. (2019). Deloitte Digital Quantifies Human Values. US: Deloitte Digital.

[8] Burnette, K. (2019). Belonging: A Conversation about Equity, Diversity, \& Inclusion.

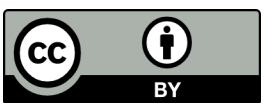

(C) 2021 by the Dr. Tom C. Hogan, Sean Gallagher, Nathan Ousey, Ray Schmitt. Submitted for possible open access publication under the terms and conditions of the Creative Commons Attribution $(\mathrm{CC}$ $\mathrm{BY})$ license 